\title{
Author Correction: Relieving ER stress to target NASH-driven hepatocellular carcinoma
}

\section{Saskia Reibe and Mark A. Febbraio}

Nature Reviews Endocrinology (2018) https://doi.org/10.1038/s41574-018-0145-7

Published online 14 December 2018

In the original version of this manuscript, the incorrect names for two proteins were given. S1P and S2P should have been defined as site-1 protease and site-2 protease. This has been corrected in the HTML and PDF versions of the manuscript.

https://doi.org/10.1038/s41574-019-0154-1 I Published online 5 January 2019 